\title{
A case of Hashimoto's encephalopathy presenting with seizures and cognitive impairment
}

\author{
Xing-Yong Chen, Yin-Zhou Wang, Hui-Xin Lei, Xu Zhang \\ Department of Neurology, Fujian Provincial Hospital, Fujian Medical University Shengli Clinical College, Fuzhou 35000, Fujian, China.
}

\section{A B S T R A C T}

\begin{abstract}
Hashimoto's encephalopathy $(\mathrm{HE})$ is a rare disease with unknown pathogenesis. An epileptic seizure is reported in association with $\mathrm{HE}$. Here, the author reported an 18-year-old girl with a history of hyperthyroidism for one year. She was admitted to the hospital due to status epilepticus. Serum thyroid function test showed that the concentration of anti-thyroid peroxidase antibodies and thyroglobulin antibody were significantly elevated. Brain magnetic resonance imaging showed that multiple abnormalities varied from bilateral frontal, parietal, occipital-temporal lobe to cerebellum hemisphere. The patient's symptoms were significantly relieved after methylprednisolone therapy. At 3-month follow-up visits, she had been symptom free. HE is a diagnosis of exclusion and should be considered when evaluating a patient with cognitive dysfunction and high titers of anti-thyroid antibodies as it responds dramatically to steroids.
\end{abstract}

Key words: Hashimoto's encephalopathy; anti-thyroid antibodies; steroid responsive encephalopathy

\section{INTRODUCTION}

Hashimoto's encephalopathy (HE), also termed as "steroid-responsive encephalopathy associated with autoimmune thyroiditis", is a rare immune-mediated encephalopathic event which affects children and adolescents. It is characterized by altered mental status, seizures, and cognitive dysfunction. It was reported seizures happened in $66 \%$ of $\mathrm{HE}$, among which status epilepticus in $12 \% \cdot{ }^{[1]}$ We reported a young female patient with generalized, tonic-clonic seizures and cognitive impairment changes who responded well to steroid treatment.

\section{CASE REPORT}

An 18-year-old girl who suffered from status epilepticus was admitted to the hospital. She had a history of hyperthyroidism for one year, and was treated with propylthiouracil (50 mg/day). There was no family history of psychiatric diseases, seizures, or other problems. Her initial vital signs were stable. On physical examination, she was conscious, cooperative, and oriented to person and time. However, she had short-term memory and computing power loss [the mini-mental state examination (MMSE) score was

Corresponding Author: Dr. Xu Zhang, Department of

Neurology, Fujian Provincial Hospital, Fujian Medical University Shengli Clinical College, Fuzhou 350001, Fujian, China.

E-mail: zhangxufjsl@163.com

\begin{tabular}{|l|l|}
\hline \multicolumn{2}{|c|}{ Access this article online } \\
\hline Quick Response Code: & Website: \\
\hline & www.nnjournal.net \\
\cline { 2 - 3 } & Dol: 10.20517/2347-8659.2015.57 \\
\hline
\end{tabular}

22, which was lower than normal] as well as bilateral limbs dystaxia. The white blood cell count was $7.55 \times$ 10\%/L, hemoglobin $156 \mathrm{~g} / \mathrm{L}$, and C-reactive protein 0.09 $\mathrm{mg} / \mathrm{dL}$. Routine biochemical analyses of liver, renal, and blood glucose were all within normal limits. The serum sodium was $131 \mathrm{mmol} / \mathrm{L}$, potassium $3.3 \mathrm{mmol} / \mathrm{L}$, and chlorine $91 \mathrm{mmol} / \mathrm{L}$. The cerebrospinal fluid (CSF) analysis displayed normal pressure $\left(145 \mathrm{mmH}_{2} \mathrm{O}\right)$ and a normal cell count and protein and glucose levels. The serum and CSF of TORCH [toxoplasmosis, other (viruses), rubella cytomegalovirus, herpes (simplex viruses)] as well as the serum rapid plasma regain test, anti-human immunodeficiency virus antibody, epatitis B surface antigen and antineutrophil cytoplasmic antibody were negative. The plasma concentrations of sex hormone and cortisol were normal. Serum thyroid function test showed that triiodothyronine (T3) $<0.300 \mathrm{nmol} / \mathrm{L}$ (reference intervals $1.30-3.10$ $\mathrm{nmol} / \mathrm{L}$ ), free triiodothyronine (FT3) $<0.400 \mathrm{pmol} / \mathrm{L}$ (reference intervals 3.10-6.80 pmol/L), upersensitive thyroidstimulating hormone (S-TSH) $0.01 \mathrm{mIU} / \mathrm{L}$ (reference intervals $0.27-4.20 \mathrm{mIU} / \mathrm{L}$ ), anti-thyroid peroxidase antibodies (TPOAb) $>600.0 \mathrm{IU} / \mathrm{mL}$ (reference intervals $0.00-34.00 \mathrm{IU} / \mathrm{L}$ ), thyroglobulin antibody (TGAb) 2,189.00 IU/mL (reference intervals 10.00-115.00 IU/mL). Electroencephalography showed a marked slowing of background rhythm as an indicator of encephalopathy but no activity corresponded with

This is an open access article distributed under the terms of the Creative Commons Attribution-NonCommercial-ShareAlike 3.0 License, which allows others to remix, tweak, and build upon the work non-commercially, as long as the author is credited and the new creations are licensed under the identical terms.

\section{For reprints contact: service@oaepublish.com}

How to cite this article: Chen $X Y$, Wang $Y Z$, Lei HX, Zhang X. A case of Hashimoto's encephalopathy presenting with seizures and cognitive impairment. Neuroimmunol Neuroinflammation 2016;3:117-9.

Received: 18-12-2015; Accepted: 25-02-2016 


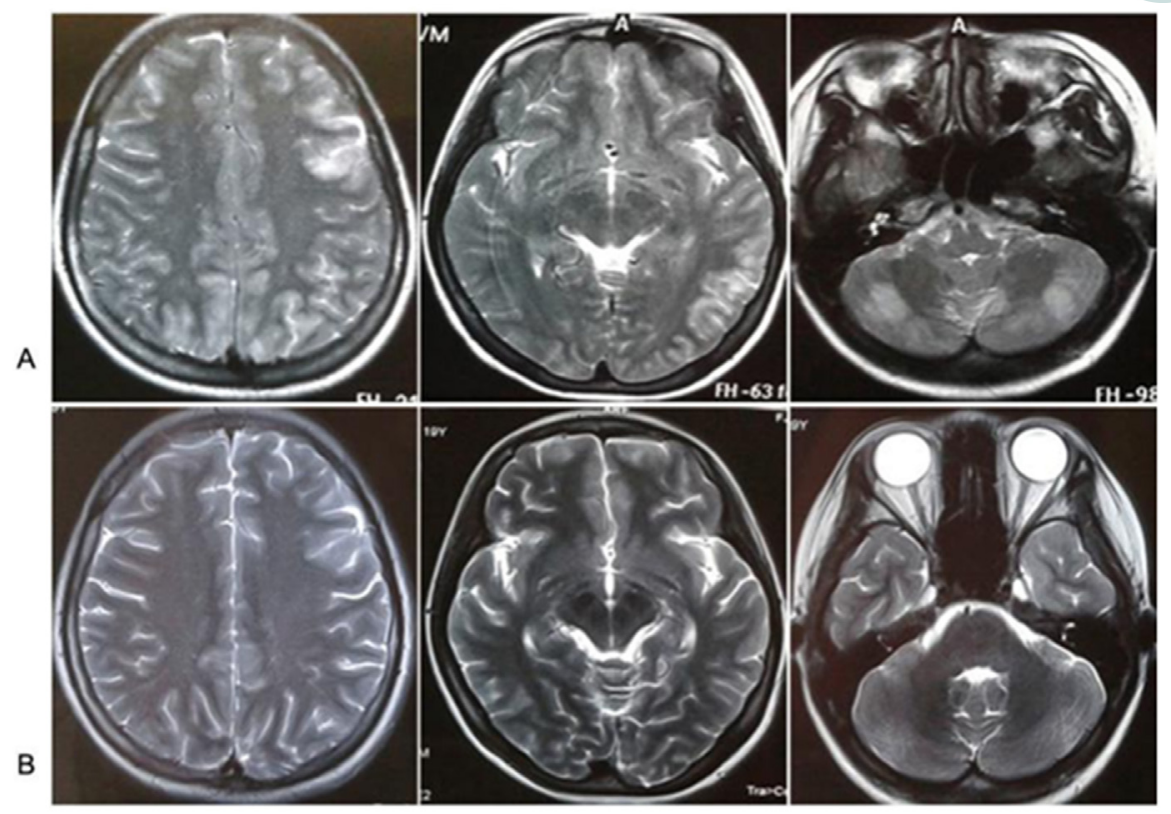

Figure 1: Brain magnetic resonance images (MRI) showed that multiple abnormalities varied from bilateral frontal, parietal, occipital-temporal lobe to cerebellum hemisphere on axial T2-weighted images (T2WI). Multiple abnormalities disappeared after steroids therapy. (A) MRI of the brain obtained before steroids therapy; (B) MRI of the brain obtained after steroids therapy

seizures. The color Doppler ultrasound examination of Thyroid and abdomen was normal. Results of CT scan of the head were unremarkable. Brain magnetic resonance images (MRI) showed that multiple abnormalities varied from bilateral frontal, parietal, occipital-temporal lobe to cerebellum hemisphere on axial T2-weighted images (T2WI) [Figure 1A]. The anticonvulsant, Tegretol (100 mg bid) was given to control the seizures. Given the indications for HE, the patient was firstly treated with intravenous injection of Dexamethasone $10 \mathrm{mg} /$ day for 7 days, followed by $5 \mathrm{mg} /$ day for 7 days. Finally, she was given with oral methylprednisolone $12 \mathrm{mg} /$ day. Her symptoms improved siginificantly and neuropsychiatric symptoms fully resolved. Retest brain MRI showed that initial multiple abnormalities disappeared compared with initial brain MRI [Figure 1B]. Serum thyroid function test showed that T3 $1.22 \mathrm{nmol} / \mathrm{L}$, FT3 $2.65 \mathrm{pmol} / \mathrm{L}, \mathrm{S}-\mathrm{TSH} 4.17 \mathrm{mIU} / \mathrm{L}$, TPOAb $369.80 \mathrm{IU} / \mathrm{mL}$, TGAb 1,102.00 IU/mL. On the 17th day, she was discharged on tapered doses of oral methylprednisolone. On 3-month follow-up visits as an outpatient, she had been symptom free without any seizure and MMSE score was 30.

\section{DISCUSSION}

As a rare steroid responsive neuropsychiatric syndrome, $\mathrm{HE}$ is associated with the serologic evidence of anti-thyroid antibodies when other causes of encephalopathy are excluded. The clinical manifestations of HE include cognitive impairment, various types of epileptic seizures, dystaxia and tremor, sleep disturbance and headache. ${ }^{[2]}$ In this case, this female patient suffered from four generalized, tonic-clonic seizures and mild cognitive impairment as well as limbs dystaxia. Recently, HE has received extensive attention due to its treatability and unclear pathogenesis. In China, Hashimoto's encephalopathy is still not fully recognized because of its complex clinical manifestions and absence of specific biomarkers. The previous research demonstrated that cognitive impairment (84.6\%) and psychiatric symptoms (38.5\%) were the most frequent symptoms, however, seizures (30.8\%) and myoclonus (7.7\%) were relatively infrequent in thirteen consecutive patients with HE. ${ }^{[3]}$ Therefore, presenting symptoms of HE may be quite variable.

The diagnosis of HE should be considered in patients presenting with the characteristic neuropsychiatric manifestations excluding other causes of encephalopathy. Generally, high levels of anti-thyroid antibodies in serum or CSF are important and helpful in the diagnosis of HE. They have no alteration in the CSF and/or imaging tests compatiblewith infectious, vascular, or neoplastic etiology, and response well to immunosuppressive therapy. ${ }^{[4]}$ Non-specific electroencephalogram abnormalities are presented in the vast majority of patients, and brain MRI may display abnormalities in 49\%, such as cerebral atrophy, focal cortical abnormality, diffuse subcortical abnormality and non-specific subcortical focal white matter abnormality. ${ }^{[5]}$ In this female patient, the analysis of CSF as well as serum inflammation biomarkers was normal, indicating the exclusion of intracranial infection. Although the elevated CSF protein is common in $\mathrm{HE}$, this change depends on the severity of the illness. Her brain MRI showed that multiple abnormalities varied from bilateral frontal, parietal, occipital-temporal lobe to cerebellum hemisphere. Neuroimaging results have no reliable diagnostic value in $\mathrm{HE}$.

In the context of the typical clinical picture, high titres of antithyroid antibodies, in particular TPOAb, are diagnostic. ${ }^{[6]}$ Recently, Blanchin et $a .^{[7]}$ reported that TPOAb from Hashimoto's encephalopathy patients 
could bind cerebellar astrocytes in HE patients but not in Hashimoto thyroiditis patients. This may support the role of TPOAb in the pathogenesis of Hashimoto's encephalopathy. TPOAb is present in $95-100 \%$ and TGAb in $73 \%$ of patients with HE. Elevated serum level of TPOAb may be related with vasculitic type Hashimoto's encephalopathy and elevated serum levels of TPOAb and TGAb may be with diffuse progressive type of $\mathrm{HE}$. However, the elevated titres of these antibodies can be tested in the healthy population. Therefore, the role of those antibodies and their pathophysiology are unknown. In addition, corticosteroid treatment is successful in most cases, which can further support the diagnosis of HE. Furthermore, other common causes of encephalopathy should be ruled out, such as intracranial infection, metabolic disease, electrolyte imbalance, poisoning or toxins, neoplasm, and the central nervous system involvement of vasculitic syndromes. With the aid of a detailed medical history and related auxiliary examination (such as CSF and MRI), it is easy to rule out these diseases.

Regarding treatment for HE, the patient's symptom improved significantly and rapidly after initiation of corticosteroid treatment, and eventually achieved a long-term stable remission. Clinical improvement with corticosteroid therapy is usually observed in the first 4-6 weeks. This positive response has been considered to be part of the definition of HE, but does not occur in all patients. ${ }^{[8]}$ Other therapies such as plasmapheresis and immunosuppressant medications have been successfully used in patients non-responsive to corticosteroids. It was reported that only a few HE patients have been treated with Intravenous immunoglobulins. ${ }^{[9]}$ Moreover, since the antithyroid antibodies could not been used as relapse markers of encephalopathys, the question of the continuation of the immunomodulatory of immunosuppressive drugs remains an open debate. ${ }^{[10]}$

In conclusion, $\mathrm{HE}$ is a rare disease associated with encephalopathy and autoimmune thyroiditis. Our research suggests that a high degree of suspicion is necessary to diagnose $\mathrm{HE}$, especially in those patients with high levels of antithyroid antibodies and presented with unexplained encephalopathy, such as seizures and cognitive dysfunction, as in our case. Corticosteroid treatment is successful for HE in most cases, however, clinicians should be aware that relapses can occur early or even late after tapering of steroid use; therefore, a long follow-up period should be recommended.

\section{Financial support and sponsorship}

This study was supported by the grants from the Fujian Province Natural Science Fund (2013J01275, 2014J01401).

\section{Conflicts of interest}

There are no conflicts of interest.

\section{REFERENCES}

1. Chong JY, Rowland LP, Utiger RD. Hashimoto encephalopathy: syndrome or myth? Arch Neurol 2003;60:164-71.

2. Castillo P, Woodruff B, Caselli R, Vernino S, Lucchinetti C, Swanson J, Noseworthy J, Aksamit A, Carter J, Sirven J, Hunder G, Fatourechi V, Mokri B, Drubach D, Pittock S, Lennon V, Boeve B. Steroid-responsive encephalopathy associated with autoimmune thyroiditis. Arch Neurol 2006;63:197-202.

3. Wang J, Zhang J, Xu L, Shi Y, Wu X, Guo Q. Cognitive impairments in Hashimoto's encephalopathy: a case-control study. PLos One 2013;8:e55758.

4. Philip R, Saran S, Gutch M, Gupta K. An unusual presentation of Hashimoto's encephalopathy. Indian $J$ Endocrinol Metab 2014;18:113-5.

5. Kothbauer-Margreiter I, Sturzenegger M, Komor J, Baumgartner R, Hess CW. Encephalopathy associated with Hashimoto thyroiditis: diagnosis and treatment. J Neurol 1996;243:585-93.

6. Mocellin R, Walterfang M, Velakoulis D. Hashimoto's encephalopathy: epidemiology, pathogenesis and management. CNS Drugs 2007;21:799-811.

7. Blanchin S, Coffin C, Viader F, Ruf J, Carayon P, Potier F, Portier E, Comby E, Allouche S, Ollivier Y, Reznik Y, Ballet JJ. Anti-thyroperoxidase antibodies from patients with Hashimoto's encephalopathy bind to cerebellar astrocytes. $J$ Neuroimmunol 2007;192:13-20.

8. Nakagawa H, Yoneda M, Fujii A, Kinomoto K, Kuriyama M. Hashimoto encephalopathy presenting with progressive cerebellar ataxia. BMJ Case Rep 2009;2009.

9. Jacob S, Rajabally YA. Current proposed mechanisms of action of intravenous immunoglobulins in inflammatory neuropathies. Curr Neuropharmacol 2009;7:337-42.

10. Visee H, Mabiglia C, Vanderaspoilden V, Gazagnes MD, Glibert G. Recurrent status epilepticus associated with Hashimoto's encephalopathy. Epilepsy Behav Case Rep 2013;1:113-7. 